\title{
Guía para la implementación de NTC ISO 9001:2008, NTC ISO 14001:2004 y NTC OHSAS 18001:2007, basada en los hallazgoos de las auditorías de certificación realizadas por el ICONTEC entre junio de 2012 y junio de 2015
}

Guide for the implementation of NTC ISO 9001:2008, NTC ISO 14001:2004 and NTC OHSAS 18001:2007, based on the findings of certification audits carried out by ICONTEC between june 2012 and june 2015

Guia para a implementação do NTC ISO 9001:2008, NTC ISO 14001:2004

e NTC OHSAS 18001:2007, com base nas conclusões das auditorias de certificação realizadas pelo ICONTEC entre junho de 2012 e junho de 2015

Recibido: 14 de febrero de 2017 Revisado: 31 de mayo de 2017 Aceptado: 30 de junio de 2017

Nicolai Abreo Rojas**

Profesional Gestión de la Prestación del Servicio de Educación ICONTEC

Nelson Ernesto Pinzón Rodríguez****

Profesional de Educación ICONTEC

\footnotetext{
* Artículo de resultado de investigación. DOI: http://dx.doi.org/10.15332/s2145-1389.2017.0002.09

** Magíster en Calidad y Gestión Integral. Especialista en Sistemas de Gestión Integrada de Calidad, Medio Ambiente y Riesgos Laborales. Ingeniero Químico. Docente tutor en Administración de Auditoría de Calidad Virtual. Profesional de Educación en ICONTEC. Correos electrónicos: nabreo@icontec.org nicolai.abreo@gmail.com

***** Magíster en Calidad y Gestión Integral (c) Especialista en Ingeniería de la Calidad y del Comportamiento. Ingeniero Industrial. Docente interno en ICONTEC. Correo electrónico: nedipaca@yahoo.es
} 


\section{RESUMEN}

La presente investigación tiene como propósito orientar a las organizaciones, a través de una guía, en la identificación de los aspectos más relevantes a tomar en cuenta para asegurar la implementación, mantenimiento y mejora de la eficacia de los sistemas de gestión de la calidad, gestión ambiental y seguridad y salud ocupacional, basados en las normas ISO 9001:2008, ISO 14001:2004 y OHSAS 18001:2007. La información obtenida y analizada en la investigación, como elemento de entrada para la elaboración de la guía, corresponde a los informes de auditorías de tercera parte realizadas por el ICONTEC en el período de tiempo comprendido entre junio de 2012 y junio de 2015. La intención investigativa fue capitalizar las experiencias de las empresas con sistemas de gestión consolidados, a través de sus hallazgos de auditoría (no conformidades y aspectos por mejorar), en favor de empresas que se encuentran en cualquier etapa de implementación, con el fin de encontrar los elementos de gestión, y en algunos casos herramientas prácticas, que permitan una administración más eficaz. Se identificaron los temas de gestión que con mayor frecuencia presentaron desviaciones negativas, resultados que se pueden deber a varios factores como: rotación en las organizaciones de personal, errores de planificación de los procesos y actividades, falta de seguimiento o baja competencia de los auditores internos, pero los resultados muestran que existe dificultad en el aseguramiento de la implementación por parte de los responsables de estos aspectos, aún en sistemas de gestión maduros.

Palabras clave: sistemas de gestión integrada, implementación de sistemas de gestión, sistemas integrados de gestión, eficacia de sistemas de gestión, auditoría, HSEQ.

\section{ABSTRACT}

The present research aims to guide the organization, through a guide, in identifying the most relevant take into account to ensure the implementation, maintenance and improvement of the effectiveness of quality management systems, environmental management and safety and occupational health, based on the ISO 9001:2008, ISO 14001:2004 and OHSAS 18001:2007 standards. The information obtained and analyzed in the research, as an input element for the preparation of the guide, corresponds to the third party audits conducted by ICONTEC in the period between June 2012 and June 2015. The investigative intention is to capitalize on the experiences of companies with consolidated management systems, through their audit findings, in favor of the companies that are in any stage of implementation, in order to find the elements of management, and in some cases of practical tools, that allow a more effective administration. The management issues that most frequently presented negative deviations were identified, results that may be due to several factors such as: turnover in personnel organizations, errors in the planning of processes and activities, lack of follow-up or low competence of internal auditors, but the results show that there is difficulty in ensuring implementation by those responsible for these aspects, even in mature management systems.

Keywords: systems of integrated management, implementation of management systems, effectiveness, audit, HSEQ.

\section{RESUMO}

O presente estudo destina-se a guiar às organizações através de uma guia, na identificação dos aspectos mais 
importantes a ter em conta para assegurar a implementação, manutenção e melhoria da eficácia dos sistemas de gestão de qualidade, gestão ambiental e segurança e saúde ocupacional, baseado sobre as normas ISO 9001:2008, ISO 14001:2004 e OHSAS 18001:2007. A informação obtida e analisada na pesquisa, como princípio para a elaboração da guia, corresponde aos relatórios de auditorias de terceira parte realizadas pelo ICONTEC no período entre junho de 2012 e junho de 2015. A intenção da pesquisa foi reunir às experiências das empresas com sistemas de gestão estabelecidos, através de seus resultados de auditoria (inconformidades e aspectos a melhorar), a favor de empresas que estão em qualquer fase da implementação, a fim de encontrar os elementos de gestão e em alguns casos, ferramentas práticas, permitindo uma administração mais eficaz. Identificados os problemas de gestão que mais frequentemente apresentaram desvios negativos, resultados que podem ser devido a vários fatores, tais como: rotação de pessoal, erros de planejamento dos processos e atividades, falta de acompanhamento, baixa competência dos auditores internos, mas os resultados mostram que há dificuldade em assegurar a implementação por parte dos responsáveis destes aspectos, mesmo em sistemas de gestão aplicados a muito tempo.

Palavras-chave: sistemas de gestão integrada, implementação de sistemas de gestão, sistemas integrados de gestão, eficácia de gestão, auditoria, HSEQ.

\section{INTRODUCCIÓN}

En el desarrollo y evolución de los sistemas de gestión y su implementación (Nunhes, Motta y de Oliveira, 2016) en las organizaciones, se plantean retos (Bernardo, Casadesus, Karapetrovic y Heras, 2012) considerables a la hora de desarrollar metodologías y herramientas eficaces para implementar en las organizaciones los requisitos. En la planificación de la implementación, la propia implementación, el mantenimiento y mejora de los sistemas de gestión, se presentan dificultades en las organizaciones que retrasan el logro de los propósitos y, en últimas, el mismo logro de los propósitos organizacionales. En este sentido, para disminuir la incertidumbre en la planificación y para generar los controles desde un principio, que eviten los problemas más frecuentes en las organizaciones y así obtener los beneficios (Bernardo, Simon, Tarí y Molina-Azorín, 2015) de los sistemas de gestión, ISO 9001, ISO 14001 y OHSAS 18001, se hace necesario empezar a explorar y capitalizar las dificultades detectadas por auditores independientes, como resultado de las auditorías de tercera parte, y así, conocer las posibles dificultades futuras, los riesgos en la implementación, mantenimiento y mejora de los sistemas de gestión de la calidad, seguridad y salud ocupacional y ambiental. Con conocimiento de las debilidades frecuentes, a través de una guía de implementación, se pueden emprender procesos más eficaces desde las primeras etapas del establecimiento de un sistema de gestión integrado SGC ISO 9001, SGA ISO 14001 y SG SYSO OHSAS 18001 (ISO, 2016; ICONTEC, 2007; ICONTEC, 2012; ICONTEC, 2015a; ICONTEC, 2015b). Esta investigación busca capitalizar la experiencia de organizaciones certificadas, con sistemas de gestión maduros, a través de los hallazgos de auditoría de tercera parte, para generar información de entrada para los proyectos de implementación, mantenimiento y mejora; construyendo así, con base en los hallazgos, una guía de implementación.

La investigación tiene otro propósito, para una parte interesada relevante, en los docentes de sistemas de gestión, que orientan la comprensión de los requisitos de los referenciales, quienes podrán enriquecer su conocimiento frente al comportamiento, en la realidad, de los enfoques de las normas y, en especial, de las dificultades para las organizaciones frente a la imple- 
mentación, mantenimiento y mejora de algunos aspectos normativos. Finalmente, el tercer grupo de interés de la presente guía es el de los auditores, de primera, segunda y tercera parte, quienes tendrán información relevante para ahondar y orientar sus auditorías, incluyendo dentro de la planificación de sus auditorías, basadas en riesgos, los resultados que esta investigación ha ubicado como temas de debilidad frente a los sistemas de gestión o barreras en su consolidación como verdaderos sistemas (Ackoff, 1978).

La guía tiene como base la experiencia de los autores y la metodología establecida en publicaciones sobre esta temática. La búsqueda de un camino más eficiente para el abordaje de tres temas relevantes: calidad, medio ambiente y seguridad y salud ocupacional, deberá ser la motivación para consolidar los beneficios (Bernardo et al., 2015) para las empresas de los tres sistemas de gestión.

Se debe considerar que los referenciales evaluados fueron: ISO 9001:2008, ISO 14001:2004 y OHSAS 18001:2007, pues eran las versiones vigentes en la ventana de investigación, sin embargo, la guía fue estructurada con base en orientaciones que incluyeran los requisitos en versiones 2015 para ISO 9001 e ISO 14001. Por otro lado, frente a OHSAS 18001, se tomaron los enfoques para la versión 2007, dado que para el momento del desarrollo de la guía no se contaba con otro referencial vigente.

\section{METODOLOGÍA}

El método utilizado fue el inductivo, partiendo de la base de los datos puntuales de hallazgos de auditoría de tercera parte, para llegar a conclusiones que puedan aplicarse a las organizaciones.

El tipo de investigación es mixto, cuantitativo y cualitativo (Hernández Sampieri, 2006), por un lado se cuantificaron las no conformidades agrupándolas por temas específicos, relacionándolas con los elementos de gestión, y por otro lado, la parte cualitativa, aplicada en la determinación de los elementos de gestión relacionados con los aspectos por mejorar, con el fin de desarrollar un análisis más práctico de los elementos que con mayor frecuencia pueden tener brechas.

La estructura de la metodología es la siguiente:

- Determinación de la muestra

- Diseño del instrumento para el registro de datos y el análisis posterior

- Análisis de los aspectos por mejorar

- Determinación de frecuencias por temas

- Análisis de resultados

- Desarrollo de la guía

Para la determinación de la muestra se tomó como base, por conveniencia, la información que tiene el ICONTEC, de los informes de auditoría de tercera parte, tanto de seguimiento como de renovación. Este filtro se aplicó con el fin de reducir la dispersión de datos en cuanto a temas de bajo impacto que eventualmente se pueden encontrar en organizaciones que hacen por primera vez, o en etapas tempranas de sus sistemas de gestión, auditorías de otorgamiento. En este sentido se tomaron organizaciones que hasta el momento de la investigación tuviesen 3 sistemas de gestión certificados, por más de 5 años consecutivos ininterrumpidos. Con este filtro esperamos de los resultados, encontrar las debilidades que con mayor frecuencia se presentan en este perfil de organizaciones. Con base en esta muestra se realizó un análisis de Pareto para determinar sectores que con mayor frecuencia presentaron debilidades y de allí se escogieron los informes que serían objeto de análisis en detalle. 
La muestra se escogió de la siguiente forma:

Primer filtro, informes de auditoría de seguimiento y renovación de las empresas certificadas ISO 9001, ISO 14001 y OHSAS 18001, en el período comprendido de junio de 2102 a junio de 2015.

Se seleccionaron de ellas las dos últimas auditorías (seguimiento y renovación) en la ventana temporal de análisis, con el fin de considerar la información que es más reciente y por lo tanto tiene más vigencia.

Se seleccionaron los sectores más representativos.

El tamaño del universo fue de 197 informes de auditoría de 65 organizaciones y de acuerdo con el análisis de frecuencias de los sectores representados, según la clasificación de IAF para ISO 9001, se escogieron estos sectores:

Tabla 1. Clasificación IAF de los sectores escogidos para el estudio.

\begin{tabular}{|cc}
\hline Código & Descripción IAF \\
\hline CS 14-0 & Productos de caucho y de plástico \\
\hline CS 19-0 & Equipo eléctrico y óptico \\
\hline CS 3-0 & Productos alimentarios, bebidas y tabaco
\end{tabular}

Fuente: elaboración propia.

18 informes de auditoría de 6 empresas de 3 sectores diferentes. Con base en ello se analizaron un total de 220 hallazgos, 40 no conformidades y 180 aspectos por mejorar.

\section{RESULTADOS Y DISCUSIÓN}

Con base en la aplicación de la herramienta para recolección de la información, se elaboraron 3 tablas, una para cada referencial, para analizar los resultados consolidados. En cada tabla se muestra el comportamiento de los hallazgos (no conformidades y aspectos por mejorar) frente a los numerales de cada norma. Se establecieron así las frecuencias de no conformidades y aspectos por mejorar frente a cada numeral de la norma y los porcentajes acumulados de hallazgos (en la columna derecha de cada tabla). Con el fin de concentrarnos en los más frecuentes se realizó corte en el 70 $\%$ del acumulado. Estos resultados se presentan en las Tablas 2, 3 y 4.

Tabla 2. Comportamiento de los hallazgos para ISO 9001:2008.

\begin{tabular}{|c|c|c|c|c|c|c|}
\hline ISO 9001 & $\begin{array}{c}\text { No } \\
\text { conformidades } \\
\text { menores }\end{array}$ & $\begin{array}{c}\text { No } \\
\text { conformidades } \\
\text { mayores }\end{array}$ & $\begin{array}{l}\text { Aspectos } \\
\text { por mejorar }\end{array}$ & $\begin{array}{l}\mathrm{NC}+ \\
\mathrm{AM}\end{array}$ & $\%$ & $\begin{array}{c}\% \\
\text { acumulado }\end{array}$ \\
\hline 8.2.2 Auditoría interna & 2 & 0 & 15 & 17 & 7,7 & 7,7 \\
\hline $\begin{array}{l}\text { 6.2.2 Competencia, formación y toma de } \\
\text { conciencia }\end{array}$ & 2 & 0 & 14 & 16 & 7,3 & 15,0 \\
\hline 4.2.3 Control de los documentos & 0 & 0 & 14 & 14 & 6,4 & 21,4 \\
\hline 6.3 Infraestructura & 2 & 0 & 12 & 14 & 6,4 & 27,7 \\
\hline $\begin{array}{l}\text { 8.5 Mejora } \\
\text { 8.5.1 Mejora continua }\end{array}$ & 0 & 0 & 14 & 14 & 6,4 & 34,1 \\
\hline
\end{tabular}


Nicolai Abreo Rojas, Nelson Ernesto Pinzón Rodríguez

\begin{tabular}{|c|c|c|c|c|c|c|}
\hline ISO 9001 & $\begin{array}{l}\text { No } \\
\text { conformidades } \\
\text { menores }\end{array}$ & $\begin{array}{c}\text { No } \\
\text { conformidades } \\
\text { mayores }\end{array}$ & $\begin{array}{l}\text { Aspectos } \\
\text { por mejorar }\end{array}$ & $\begin{array}{c}\mathrm{NC}+ \\
\mathrm{AM}\end{array}$ & $\%$ & $\begin{array}{c}\% \\
\text { acumulado }\end{array}$ \\
\hline 8.5.2 Acción correctiva & 4 & 0 & 9 & 13 & 5,9 & 40,0 \\
\hline $\begin{array}{l}\text { 7.5 Producción y prestación del servicio } \\
\text { 7.5.1 Control de la producción y de la } \\
\text { prestación del servicio }\end{array}$ & 4 & 0 & 8 & 12 & 5,5 & 45,5 \\
\hline $\begin{array}{l}\text { 7.6 Control de los equipos de seguimien- } \\
\text { to y de medición }\end{array}$ & 3 & 0 & 9 & 12 & 5,5 & 50,9 \\
\hline $\begin{array}{l}\text { 8.2 Seguimiento y medición } \\
\text { 8.2.1 Satisfacción del cliente }\end{array}$ & 1 & 0 & 8 & 9 & 4,1 & 55,0 \\
\hline 6.4 Ambiente de trabajo & 2 & 0 & 6 & 8 & 3,6 & 58,6 \\
\hline $\begin{array}{l}\text { 7.4 Compras } \\
\text { 7.4.1 Proceso de compras }\end{array}$ & 1 & 0 & 7 & 8 & 3,6 & 62,3 \\
\hline $\begin{array}{l}\text { 8.2.3 Seguimiento y medición de los } \\
\text { procesos }\end{array}$ & 2 & 0 & 5 & 7 & 3,2 & 65,5 \\
\hline $\begin{array}{l}\text { 8.2.4 Seguimiento y medición del } \\
\text { producto }\end{array}$ & 2 & 0 & 5 & 7 & 3,2 & 68,6 \\
\hline 8.5.3 Acción preventiva & 2 & 0 & 5 & 7 & 3,2 & 71,8 \\
\hline
\end{tabular}

Fuente: elaboración propia.

Frente a ISO 14001 el comportamiento de la muestra

fue el siguiente:

Tabla 3. Comportamiento de los hallazgos para ISO 14001:2004.

\begin{tabular}{|c|c|c|c|c|c|c|}
\hline ISO 14001 & $\begin{array}{c}\text { No } \\
\text { conformidades } \\
\text { menores }\end{array}$ & $\begin{array}{c}\text { No } \\
\text { conformidades } \\
\text { mayores }\end{array}$ & $\begin{array}{l}\text { Aspectos } \\
\text { por } \\
\text { mejorar }\end{array}$ & $\begin{array}{c}\mathrm{NC}+ \\
\mathrm{AM}\end{array}$ & $\%$ & $\begin{array}{c}\% \\
\text { acumulado }\end{array}$ \\
\hline 4.4.6 Control operacional & 20 & 0 & 53 & 73 & 25,8 & 25,8 \\
\hline 4.3.1 Aspectos ambientales & 3 & 0 & 29 & 32 & 11,3 & 37,1 \\
\hline 4.4.7 Preparación y respuesta ante emergencias & 9 & 0 & 18 & 27 & 9,5 & 46,6 \\
\hline 4.3.2 Requisitos legales y otros requisitos & 9 & 0 & 17 & 26 & 9,2 & 55,8 \\
\hline 4.5.1 Medición y seguimiento & 2 & 0 & 19 & 21 & 7,4 & 63,3 \\
\hline $\begin{array}{l}\text { 4.4.2 Competencia, formación y toma de } \\
\text { conciencia }\end{array}$ & 3 & 0 & 17 & 20 & 7,1 & 70,3 \\
\hline
\end{tabular}

Fuente: elaboración propia. 
Por otro lado, las empresas tuvieron este resultado con

OHSAS 18001:

Tabla 4. Comportamiento de los hallazgos para OHSAS 18001:2007.

\begin{tabular}{|c|c|c|c|c|c|c|}
\hline OHSAS 18001 & $\begin{array}{l}\text { No conformidades } \\
\text { menores }\end{array}$ & $\begin{array}{l}\text { No conformidades } \\
\text { mayores }\end{array}$ & $\begin{array}{l}\text { Aspectos por } \\
\text { mejorar }\end{array}$ & $\begin{array}{l}\mathrm{NC}+ \\
\mathrm{AM}\end{array}$ & $\%$ & $\%$ acumulado \\
\hline 4.4.6 Control operacional & 21 & 0 & 43 & 64 & 24,0 & 24,0 \\
\hline $\begin{array}{l}\text { 4.3.1 Identificación de peligros, } \\
\text { valoración de riesgos y deter- } \\
\text { minación de los controles }\end{array}$ & 5 & 0 & 27 & 32 & 12,0 & 36,0 \\
\hline $\begin{array}{l}\text { 4.4.7 Preparación y respuesta } \\
\text { ante emergencias }\end{array}$ & 7 & 0 & 18 & 25 & 9,4 & 45,3 \\
\hline $\begin{array}{l}\text { 4.4.2 Competencia, formación } \\
\text { y toma de conciencia }\end{array}$ & 5 & 0 & 17 & 22 & 8,2 & 53,6 \\
\hline $\begin{array}{l}\text { 4.5.3.2 No conformidad, acción } \\
\text { correctiva y acción preventiva }\end{array}$ & 2 & 0 & 16 & 18 & 6,7 & 60,3 \\
\hline $\begin{array}{l}\text { 4.5.1 Medición y seguimiento } \\
\text { del desempeño }\end{array}$ & 3 & 0 & 13 & 16 & 6,0 & 66,3 \\
\hline 4.5.5 Auditoría interna & 1 & 0 & 14 & 15 & 5,6 & 71,9 \\
\hline
\end{tabular}

Fuente: elaboración propia.

Con base en el análisis por cada norma se consolidaron, En la figura 1 se presentan los resultados del análisis con un enfoque integrado, los aspectos de gestión rela- $\quad$ y de la agrupación, para las tres normas, por aspecto cionados con los hallazgos más frecuentes. de gestión.

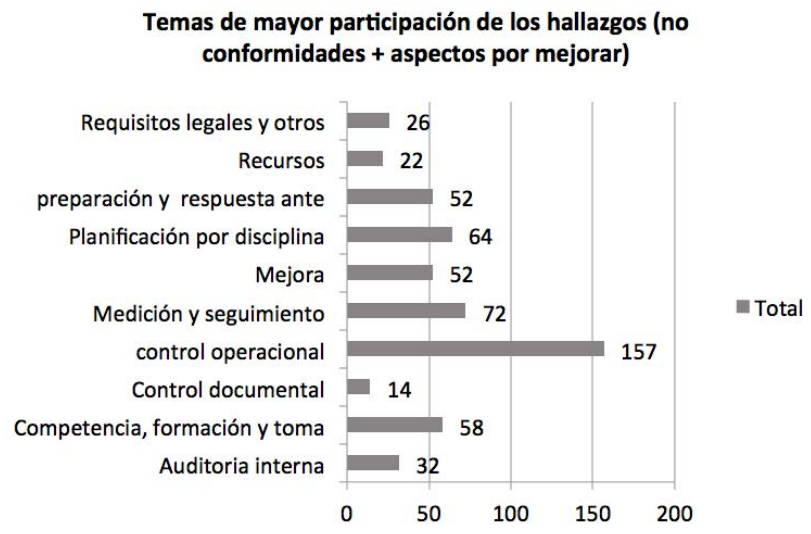

Fuente: elaboración propia.

Figura 1. Temas con mayor participación en los hallazgos para las tres normas.

Con base en los resultados se establecieron los temas que tación, para aumentar la eficacia de los sistemas de gespara cada norma es necesario robustecer en su implemen- tión, y que se ampliaron en la guía de implementación: 
Nicolai Abreo Rojas, Nelson Ernesto Pinzón Rodríguez

Tabla 4. Temas de mayor frecuencia en no conformidades y aspectos por mejorar.
Auditoría interna
4.5.5 Auditoría interna - OHSAS 18001
8.2.2 Auditoría interna - ISO 9001
Competencia, formación y toma de conciencia
4.4.2 Competencia, formación y toma de conciencia - ISO 14001
4.4.2 Competencia, formación y toma de conciencia - OHSAS 18001
6.2.2 Competencia, formación y toma de conciencia - ISO 9001

Control documental

4.2.3 Control de los documentos - ISO 9001

Control operacional

4.4.6 Control operacional - ISO 14001

4.4.6 Control operacional - OHSAS 18001

7.4 Compras

7.4.1 Proceso de compras - ISO 9001

7.5 Producción y prestación del servicio

7.5.1 Control de la producción y de la prestación del servicio - ISO 9001

Medición y seguimiento

4.5.1 Medición y seguimiento - ISO 14001

4.5.1 Medición y seguimiento del desempeño - OHSAS 18001

7.6 Control de los equipos de seguimiento y de medición - ISO 9001

8.2 Seguimiento y medición

8.2.1 Satisfacción del cliente - ISO 9001

8.2.3 Seguimiento y medición de los procesos - ISO 9001

8.2.4 Seguimiento y medición del producto - ISO 9001

\title{
Mejora
}

4.5.3.2 No conformidad, acción correctiva y acción preventiva - OHSAS 18001

8.5 Mejora

8.5.1 Mejora continua - ISO 9001

8.5.2 Acción correctiva - ISO 9001

8.5.3 Acción preventiva - ISO 9001

\section{Planificación por disciplina}

4.3.1 Aspectos ambientales - ISO 14001

4.3.1 Identificación de peligros, valoración de riesgos y determinación de los controles - OHSAS 18001

Preparación y respuesta ante emergencias

4.4.7 Preparación y respuesta ante emergencias - ISO 14001

4.4.7 Preparación y respuesta ante emergencias - OHSAS 18001

\author{
Recursos \\ 6.3 Infraestructura - ISO 9001 \\ 6.4 Ambiente de trabajo - ISO 9001 \\ Requisitos legales y otros \\ 4.3.2 Requisitos legales y otros requisitos - ISO 14001
}

Fuente: ICONTEC, Sistemas de Gestión de la Calidad. Requisitos. ISO 9001:2015, 2015. ICONTEC, Sistemas de Gestión Ambiental. Requisitos con orientación para su uso. ISO 14001:2015, 2015. ICONTEC, Sistemas de Gestión de Seguridad y Salud Ocupacional. Requisitos. OHSAS 18001:2007, 2007. 


\section{CONCLUSIONES}

Se observó en el análisis cualitativo de la información suministrada por las empresas en cada uno de los sistemas, lo siguiente:

Para NTC ISO 9001:2008. Los cinco primeros aspectos que se encontraron con mayor debilidad fueron: auditorías internas, competencia, formación y toma de conciencia, control de los documentos, e infraestructura y mejora continua,

Para NTC ISO 14001:2004. Los tres aspectos que se encontraron de mayor debilidad fueron: el control operacional, los aspectos ambientales y los requisitos legales y otros.

Para NTC OHSAS 18001:2007. Los siete aspectos que se encontraron de mayor debilidad fueron: el control operacional, identificación de peligros, valoración de riesgos y determinación de los controles, preparación y respuesta ante emergencias, competencia, formación y toma de conciencia, no conformidad, acción correctiva y acción preventiva, medición y seguimiento del desempeño y auditoría interna.

Se identificaron así los temas de gestión que con mayor frecuencia presentaron desviaciones negativas, resultados estos que se pueden deber a varios factores como: rotación en las organizaciones de personal, errores de planificación de los procesos y actividades, falta de seguimiento, baja competencia de los auditores internos, etc., pero los resultados muestran que existe dificultad en el aseguramiento de la implementación por parte de los responsables de estos aspectos, aún en sistemas de gestión maduros.

Es necesario el desarrollo de esta guía para orientar a las organizaciones e incrementar su capacidad de cumplir, bien sea en procesos de educación en temas de sistemas de gestión, o también en procesos de auditoría de primera, segunda y tercera parte, para que los auditores conozcan las posibles áreas de riesgo y puedan realizar auditorías enfocadas en los controles.

El desarrollo de la investigación llevó a identificar al control operacional, en medio ambiente y seguridad y salud ocupacional, como el aspecto de gestión con mayor potencial de mejora en las organizaciones, la implementación de controles a materiales, equipos, mercancía y proveedores tienen un impacto significativo en los resultados de los sistemas de gestión. En la norma ISO 9001 se reparten de una forma más uniforme los resultados, siendo auditoría interna la que más puntúa con 17 hallazgos encontrados.

Desarrollar, cada vez más, programa educativos en los aspectos encontrados, para lograr implementadores más competentes, más eficaces y eficientes en su labor de direccionar estos procesos.

Extender el estudio con ventanas de análisis más amplias e incluyendo factores como la competencia de los auditores, la experiencia, y eventualmente identificando áreas en que los auditores tengan debilidades, por ejemplo, en la evaluación de sistemas de gestión, en herramientas de gestión y en la revisión de planes de acción correctiva. Estos son aspectos de los que no se conoce información, pero pueden capitalizarse en beneficio de las empresas y del organismo de evaluación de la conformidad.

La formulación de la guía se basa en la estructura de alto nivel, por lo que su aplicación en la implementación de las versiones 2015 de ISO 9001 e ISO 14001, es aplicable. Las orientaciones presentadas en la guía tienen el enfoque 2015, dado que las fuentes de información fueron las guías de aplicación de AENOR y de APCER.

Se podrá continuar, por otros grupos de investigación, el estudio, y en consecuencia, complementar la guía cuando se cuente con información de evaluación de la conformidad para las versiones 2015. 


\section{REFERENCIAS}

Ackoff, R. (1978). The Art of Problem Solving. New York, NY: John Wiley y Sons Inc.

Bernardo, M., Casadesus, M., Karapetrovic, S., y Heras, I. (2012). Do integration difficulties influence management system integration levels?. Journal of Cleaner Production, 21(1), 23-33.

Bernardo, M., Simon, A., Tarí, J. J., y Molina-Azorín, J. F. (2015). Benefits of management systems integration: A literature review. Journal of Cleaner Production, 94(Supplement C), 260-267.

Hernández Sampieri, R. (2006). Metodología de la investigación. México D. F., México: McGraw-Hill Interamericana.

ISO. (2016). ISO 14004:2016(es) Sistemas de gestión ambiental. Directrices generales sobre la implementación. Ginebra, Suiza: ISO.

ICONTEC. (2007). Sistemas de Gestión de Seguridad y Salud Ocupacional. Requisitos. OHSAS 18001:2007. Instituto Colombiano de Normas Técnicas y Certificación. Bogotá D. C., Colombia: ICONTEC.

ICONTEC. (2012). Guía para la identificación de los peligros y la valoración de los riesgos en seguridad y salud ocupacional GTC 45:2012. Instituto Colombiano de Normas Técnicas y Certificación. Bogotá D. C., Colombia: ICONTEC.
ICONTEC. (2015a). Sistemas de Gestión de la Calidad. Requisitos. ISO 9001:2015. Instituto Colombiano de Normas Técnicas y Certificación. Bogotá D. C., Colombia: ICONTEC.

ICONTEC. (2015b). Sistemas de Gestión Ambiental. Requisitos con orientación para su uso. ISO 14001:2015. Instituto Colombiano de Normas Técnicas y Certificación. Bogotá D. C., Colombia: ICONTEC.

Etkin, J. (2003). Gestión de la complejidad en las organizaciones. Oxford University Press, 323-383.

García, F., Ibáñez, J., y Francisco, A. (2000). El análisis de la realidad social: métodos y técnicas de investigación. Alian«a, 64.

Gómez, J. A. (2016). Guía para la aplicación de ISO 9001:2015. Bogotá: Alfaomega Colombiana S.A. y AENOR.

Nunhes, T. V., Motta, L. C. F., y de Oliveira, O. J. (2016). Evolution of integrated management systems research on the Journal of Cleaner Production: Identification of contributions and gaps in the literature. Journal of Cleaner Production, 139, 1234-1244.

Senge, P. (2005). La quinta disciplina en la práctica. Buenos Aires, Argentina: Garnica.

Valdéz, J. L., Alonso, M. C., Calso, N., y Novo, M. (2016). Guía para la aplicación de UNE-EN ISO 14001:2015. Madrid, España: AENOR (Asociación Española de Normalización y Certificación). 